\title{
A THEORETICAL STUDY ON THE VIBRATIONAL SPECTRA OF POLYCYCLIC AROMATIC HYDROCARBON MOLECULES WITH ALIPHATIC SIDEGROUPS
}

\author{
SEyedAbdolReza SAdJAdi, Yong Zhang (張泳), ANd Sun KwoK (郭新) \\ Space Astronomy Laboratory, Faculty of Science, The University of Hong Kong, Pokfulam Road, Hong Kong, China; sunkwok@ hku.hk \\ Received 2014 August 17; accepted 2014 December 31; published 2015 February 27
}

\begin{abstract}
The role of aliphatic side groups in the formation of astronomical unidentified infrared emission (UIE) features is investigated by applying the density functional theory to a series of molecules with mixed aliphatic-aromatic structures. The effects of introducing various aliphatic groups to a fixed polycyclic aromatic hydrocarbon (PAH) core (ovalene) are studied. Simulated spectra for each molecule are produced by applying a Drude profile at $T=500 \mathrm{~K}$ while the molecule is kept at its electronic ground state. The vibrational normal modes are classified using a semi-quantitative method. This allows us to separate the aromatic and aliphatic vibrations, and therefore provides clues to what types of vibrations are responsible for the emissions bands at different wavelengths. We find that many of the UIE bands are not pure aromatic vibrational bands but may represent coupled vibrational modes. The effects of aliphatic groups on the formation of the $8 \mu \mathrm{m}$ plateau are quantitatively determined. The vibrational motions of methyl $\left(-\mathrm{CH}_{3}\right)$ and methylene $\left(-\mathrm{CH}_{2}-\right)$ groups can cause the merging of the vibrational bands of the parent PAH and the forming of broad features. These results suggest that aliphatic structures can play an important role in the UIE phenomenon.
\end{abstract}

Key words: infrared: ISM - ISM: lines and bands - ISM: molecules - planetary nebulae: general

Supporting material: animations

\section{INTRODUCTION}

While it is widely known that interstellar dust contains an aromatic component, the presence of an aliphatic component is not as well appreciated. The $3.4 \mu \mathrm{m}$ feature was first detected in absorption against the infrared background of the Galactic center (Soifer et al. 1976), but no identification was made at that time. This feature was rediscovered by Wickramasinghe \& Allen (1980) a few years later in a search for organic dust in the diffuse interstellar medium. Higher spectral resolution observations of the $3.4 \mu \mathrm{m}$ feature in the planetary nebula IRAS $21282+5050$ show that it is composed of several subfeatures at 3.4, 3.46, 3.51, and $3.56 \mu \mathrm{m}$ (Jourdain de Muizon et al. 1990), which can be identified as $\mathrm{C}-\mathrm{H}$ stretching modes of the methyl $\left(-\mathrm{CH}_{3}\right)$ and methylene $\left(-\mathrm{CH}_{2}\right)$ groups in aliphatic hydrocarbon materials (Duley \& Williams 1983). Since then, the feature has been seen in emission in protoplanetary nebulae (Geballe et al. 1992; Hrivnak et al. 2007) and in absorption in galaxies (Wright et al. 1996; Dartois et al. 2004, 2007).

In addition to the $3.4 \mu \mathrm{m}$ aliphatic $\mathrm{C}-\mathrm{H}$ stretching mode, features at 6.9 and $7.3 \mu \mathrm{m}$ corresponding to the bending modes of aliphatic methyl and methylene groups were observed as early as 1979 (Willner et al. 1979). These features have been detected in absorption (Chiar et al. 2002) as well as in emission (Kwok et al. 1999).

Akari observations of M82 have shown that the strength of the $3.4 \mu \mathrm{m}$ feature increases from the disk to the halo of the galaxy, suggesting that aliphatic organics are distributed throughout the volume of galaxies (Yamagishi et al. 2012). Since the abundance can easily be derived from the strengths of absorption features, it is estimated that the amount of carbon tied up in aliphatic compounds represents $15 \%-30 \%$ of the total interstellar carbon (Dartois 2011).

Another manifestation of the aliphatic component is the strong, broad emission plateau features seen in planetary nebulae and protoplanetary nebulae. The plateau features centered around 8 and $12 \mu \mathrm{m}$ were first seen in the IRAS LRS (Kwok et al. 1989) and Kuiper Airborne Observatory (Buss et al. 1990) spectra of protoplanetary nebulae. These two plateau features are much better defined by Infrared Space Observatory observations and have been identified as superpositions of a variety of aliphatic side groups attached to aromatic rings (Kwok et al. 2001). These broad features cannot be explained by traditional pure aromatic models such as polycyclic aromatic hydrocarbon (PAH) molecules. The presence of these features, as well as the 3.4, 6.9, and $7.3 \mu \mathrm{m}$ features, suggests that the chemical structures of organic dust in the interstellar medium are much more complex than previously believed.

By introducing $\mathrm{H}$ into graphite $\left(s p^{2}\right)$ and diamond $\left(s p^{3}\right)$, a variety of amorphous $\mathrm{C}-\mathrm{H}$ alloys can be created (Robertson 2002). Different geometric structures with long and short ranges can be created by varying the aromatic-to-aliphatic ratios. The spectral characteristics of these structures and their possible role as carriers of the unidentified infrared emission (UIE) bands have recently been extensively studied (Jones 2012a, 2012b, 2012c). Laboratory studies of such amorphous carbonaceous solids (Dischler et al. 1983a, 1983b) suggest that they show many spectral characteristics similar to astronomical UIE bands.

The vibrational properties of PAH molecules have been extensively studied (e.g., Bauschlicher \& Langhoff 1997; Ricca et al. 2013). Infrared spectra of PAHs with different geometries, sizes, and charge states have been obtained with quantum chemical calculations (Malloci et al. 2007; Bauschlicher et al. 2010; Boersma et al. 2014). Recently, there has been increased interest in the effects of aliphatic structures. The UIE bands have been suggested as being better explained by mixed aromatic/ aliphatic organic nanoparticles (MAON; Kwok \& Zhang 2011). The contributions of methyl and methylene groups have been computed by Yang et al. (2013), and the vibrational spectra of a variety of aliphatic structures have been calculated by Papoular \& Papoular (2014).

Through advances in computational chemistry methods and the increasing power of computing hardware, it is now possible to perform a theoretical investigation of the vibrational 
properties of large organic molecules (Papoular 2013; Ricca et al. 2013). With such theoretical studies, we can identify the explicit roles of aliphatic groups in the vibrational spectrum of the molecules. In this work, we aim to study the changes in the infrared emission spectra of a PAH molecule upon addition of the aliphatic groups and to specifically identify the types of vibrational motions responsible for such changes.

\section{COMPUTATIONAL METHODS}

Our calculations are based on the density functional theory (DFT; Hohenberg \& Kohn 1964; Kohn \& Sham 1965; Ziegler 1991) where the molecular non-relativistic Schrödinger equation is solved under the Born-Oppenheimer approximation (Born \& Oppenheimer 1927). The BH\&HLYP hybrid functional (Becke 1993) in combination with polarization consistent basis set PC1 (Jensen 2001, 2002) are applied to obtain the equilibrium geometries and the fundamental vibrational frequencies of hydrocarbon molecules. The chosen PC1 basis set includes the necessary polarized $d$ and $p$ orbitals on $\mathrm{C}$ and $\mathrm{H}$ centers individually. The accuracy of the vibrational frequencies under the BH\&HLYP/PC1 model is estimated to have an rms error of $2 \mathrm{~cm}^{-1}$ (Laury et al. 2012). All geometries have been optimized under the default criteria, i.e., forces $<4.5 \times 10^{-4}$ hartree/bohr and $\mathrm{rms}$ error of the forces less than $3.0 \times 10^{-4}$. A fine integration grid, combined with the default convergence criterion of $1 \times 10^{-8}$, is applied for self-consistent field calculation of the DFT density matrix. The optimized geometries are all characterized as local minima, established by the positive values of all frequencies and their associated eigenvalues of the second derivative matrix. The vibrational frequencies are calculated via the harmonic normal mode approximation (Ochterski 1999). The scaling factors of 0.9311 and 0.9352 for vibrational frequencies $>1000 \mathrm{~cm}^{-1}$ and $<1000 \mathrm{~cm}^{-1}$, respectively, are then applied (Laury et al. 2012). The final infrared spectra for each isomer are constructed by plotting the scaled frequency values versus the calculated vibrational mode intensities.

The simple quantitative decomposition normal mode analysis is adopted in combination with customary visualizing of the normal modes. The Cartesian displacements $x, y, z$ of all the atoms calculated in the vibrational analysis (Ochterski 1999) are converted to a quantitative measure, $R$, which is defined as

$$
R=\sqrt{x^{2}+y^{2}+z^{2}},
$$

where $x, y, z$, and $R$ are all expressed in units of $\AA$.

The analysis is performed with a small code called Vibanalysis written by SAS. This code has the ability to read the atom displacement in Cartesian coordinates and to perform the described analysis. Its output is the list of atoms (labeled as aliphatic or aromatic) with a major contribution in the corresponding normal mode, i.e., the atoms with $R \geqslant 0.1 \AA$.

All ab initio calculations have been performed by the Gaussian 09, Revision C.01 package (Frisch et al. 2009). The HKU grid-point supercomputer facility equipped with 8 and $12 \mathrm{CPU}$ cores and 30-40 GB of RAM on each node has been utilized for this purpose. Visualizing and manipulating the results of vibrational normal mode analysis were performed using the Chemcraft suit program. ${ }^{1}$

In order to facilitate comparison with the observed astronomical infrared emission spectra, the calculated vibrational-mode intensities have been converted to a simulated observed spectra

\footnotetext{
http://www.chemcraftprog.com
}

by applying a thermal excitation and a Drude profile. Assuming a thermal population, the intensities of emission lines can be calculated from the vibrational-mode intensities calculated by DFT. We assume that the intensity of the emission line at certain wavelengths is equal to its absorption intensity calculated by DFT. Ignoring the contributions from hot bands and overtones, the line intensity of the $i$ th vibrational mode $\left(I_{i}\right)$ is proportional to $\exp \left(-h v_{i} / k T\right)$, where $v_{i}$ is the frequency of the normal mode, and $h$ and $k$ are the Planck and Boltzmann constants, respectively. A Drude profile,

$$
F_{i}(\lambda)=\frac{2 I_{i} \lambda_{i}}{\pi c \gamma} \times \frac{\gamma^{2}}{\left(\lambda / \lambda_{i}-\lambda_{i} / \lambda\right)^{2}+\gamma^{2}},
$$

is applied to broaden all of the IR features, where $\lambda_{i}$ is the central wavelength, $\gamma$ is the fractional FWHM of the simulated peaks, and $c$ is the speed of light. The value of $\gamma$ is chosen to fit the experimental or observational spectra. The modeled spectra are then obtained by suming over all $F_{i}(\lambda)$ values:

$$
F(\lambda)=\Sigma F_{i}(\lambda)
$$

where $F(\lambda)$ is the final intensity of the emission band at each point on the Drude profile. In this model, no electronic excitations have been considered, and therefore all of the molecules in our present study are kept in their singlet electronic ground state. $T=500 \mathrm{~K}$ is chosen for the simulation temperature of emission spectra as this is the optimum temperature suggested by Cook \& Saykally (1998) which keeps the long wavelength vibrational levels well populated.

\section{THEORY VERSUS EXPERIMENT}

In order to check the accuracy of our quantum chemical model for predicting the frequencies and intensities of the fundamental vibrational normal modes in hydrocarbon molecules, we compare our theoretical IR spectra with recent experimental FT-IR data on number of hydrogenated PAH molecules reported by Sandford et al. (2013). These are the Group $\mathrm{H}$ molecules $\mathrm{C}_{22} \mathrm{H}_{16}$ (DHDB[ah]A), $\mathrm{C}_{20} \mathrm{H}_{14}$ (DHB[e]P), and $\mathrm{C}_{20} \mathrm{H}_{14}$ (TRIP), which are the largest molecules in the set. Qualitatively, we found very good agreement between the calculated band positions and intensity ratios and their corresponding experimental counterparts in the IR spectra. A comparison between the calculated IR spectrum with the experimental spectrum of TRIP is shown in Figure 1. We have also evaluated the accuracy of our theoretical results quantitatively and the results are shown in Table 1. The calculated spectra are broadened using the Drude function with the FWHM equal to that of the experimental spectra. Each peak is actually a composition of multiple transitions. The peak positions and integrated strengths are measured and listed in Table 1. We find that the BH\&HLYP vibrational modes intensity ratios are in very good agreement with the reported experimental band strengths. Accuracy of less than $0.2 \mu \mathrm{m}$ is achieved for the peak positions of the bands with the largest theoretical error found for the $8.8 \mu \mathrm{m}$ band in Table 1 .

The reliability of our method for simulating the infrared emission spectrum was verified against experimental spectra of PAH molecules (Cook et al. 1996). Comparisons are made with three selected PAH molecules: phenanthrene $\left(\mathrm{C}_{14} \mathrm{H}_{10}\right)$, pyrene $\left(\mathrm{C}_{16} \mathrm{H}_{10}\right)$, and coronene $\left(\mathrm{C}_{24} \mathrm{H}_{12}\right)$ (Figure 2$)$. While there are differences in relative intensities, the positions of the theoretical bands are in good agreement with experimental values. The average absolute errors of the positions of simulated bands 


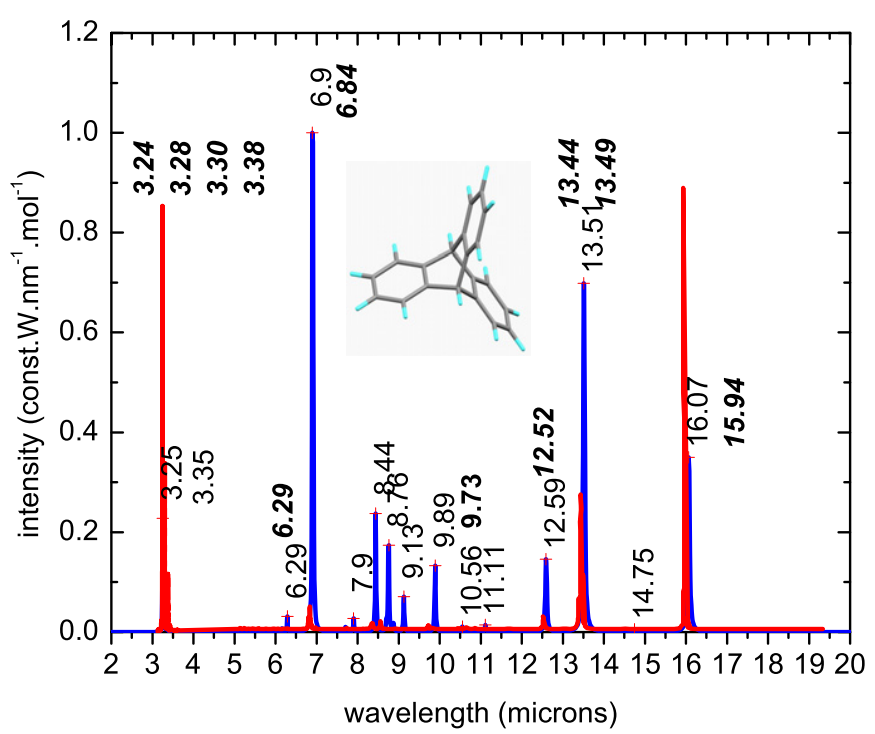

Figure 1. Simulated IR spectra of $\mathrm{C}_{20} \mathrm{H}_{14}$ (shown in blue) at $500 \mathrm{~K}$ compared with the experimental data of Sandford et al. (2013) (shown in red). The broadening parameters were chosen to match experimental broadening. The peak wavelengths of some of the features are also marked: theoretical values in normal font and experimental values in bold italics. The optimized geometry of the molecule is shown in the insert.

Table 1

The Comparison between Simulated IR Spectrum and Experimental FT-IR Data

\begin{tabular}{|c|c|c|c|c|c|}
\hline \multicolumn{3}{|c|}{ Experiment $^{\mathrm{a}}$} & \multicolumn{2}{|c|}{ DFT } & \multirow{2}{*}{$\begin{array}{c}\text { Absolute Error }{ }^{\mathrm{b}} \\
(\mu \mathrm{m})\end{array}$} \\
\hline $\begin{array}{r}\text { Posi } \\
\left(\mathrm{cm}^{-1}\right)\end{array}$ & $\begin{array}{l}\text { on } \\
(\mu \mathrm{m})\end{array}$ & Strengths ${ }^{\mathrm{c}}$ & $\begin{array}{l}\text { Position } \\
(\mu \mathrm{m})\end{array}$ & Strengths ${ }^{c}$ & \\
\hline 626.2 & 15.96 & 0.57 & 16.07 & 0.35 & 0.11 \\
\hline 742.4 & 13.47 & 1.0 & 13.51 & 0.70 & 0.04 \\
\hline 796.7 & 12.55 & 0.15 & 12.59 & 0.15 & 0.04 \\
\hline 1166.2 & 8.57 & 0.11 & 8.76 & 0.17 & 0.19 \\
\hline 1193.2 & 8.38 & 0.19 & 8.44 & 0.24 & 0.06 \\
\hline 1460.4 & 6.85 & 0.66 & 6.90 & 1.0 & 0.05 \\
\hline 2958.3 & 3.38 & 0.2 & 3.35 & 0.13 & 0.03 \\
\hline 3028.6 & 3.30 & 0.84 & 3.25 & 0.23 & 0.05 \\
\hline
\end{tabular}

Notes.

a The experimental data are the peak positions and strengths of the dominant band in each region of spectra (Sandford et al. 2013).

b $\left|\lambda_{\text {exp }}-\lambda_{\text {theo }}\right|$

c Normalized to the strongest line.

are $15.31 \mathrm{~cm}^{-1}, 20.68 \mathrm{~cm}^{-1}$, and $20.47 \mathrm{~cm}^{-1}$ (equivalent to $0.12-0.13 \mu \mathrm{m}$ error) for phenathrene, pyerene, and coronene, respectively. The $10.42 \mu \mathrm{m}$ band in experimental spectra of coronene (Figure 2) is a contamination band as reported by Cook et al. (1998).

\section{INTERPRETATION OF EMISSION SPECTRA}

The detection of astronomical aliphatic emission features has led to interest in the exploration of variations from the PAH model, which until recently has been the most widely accepted model for the astronomical UIE bands. Recent efforts include the laboratory study of partially hydrogenated PAHs (Sandford et al. 2013) and theoretical studies of the vibrational modes of kerogen derivatives (Papoular 2013). In this paper, we explore the intermediate case through the gradual addition of aliphatic side groups to an aromatic core. We begin with a medium-size PAH molecule, ovalene $\left(\mathrm{C}_{32} \mathrm{H}_{14}\right.$,

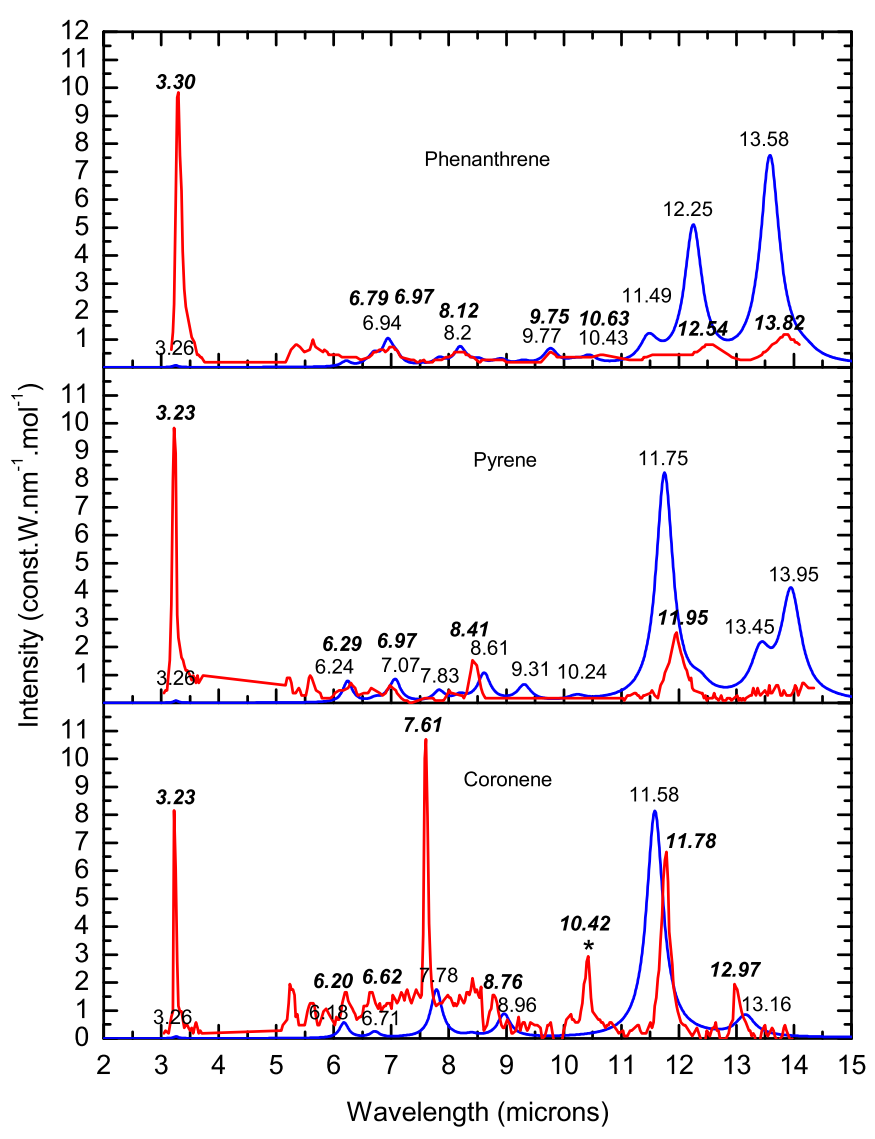

Figure 2. Comparison of the experimental spectra (in red) of phenanthrene (top panel), pyrene (middle panel), and coronene (bottom panel) (Cook et al. 1996) with our theoretical DFT spectrum (in blue). The numbers are peak wavelengths of the features with the experimental values in bold italics and the theoretical values in regular font. The * marks a contamination band in the spectrum of coronene (Cook et al. 1998). The digitized experimental spectrum intensity values have been scaled up by a factor of 10 for ease of comparison.

Figure 3), and gradually replace its peripheral $\mathrm{H}$ atoms with the different $-\mathrm{CH}=\mathrm{CHCH}_{3},-\mathrm{CH}_{2} \mathrm{CH}=\mathrm{CH}_{2},-\mathrm{CH}_{2} \mathrm{CH}_{2} \mathrm{CH}_{2}-$, $-\mathrm{CH}_{2}\left(\mathrm{CH}_{2}\right)_{3} \mathrm{CH}_{3},-\mathrm{C}\left(\mathrm{CH}_{3}\right)_{3}$, and $-\mathrm{CH}=\mathrm{C}\left(\mathrm{CH}_{3}\right)_{2}$ aliphatic functional groups. Such structures are similar to those described in Figure 4 of Kwok et al. (2001). With different types of functional groups the symmetry of the molecule is reduced to $C_{1}$, and therefore the number of IR active modes is maximized. The chemical formula, two-dimensional (2D) structural formula, and three-dimensional (3D) ball and stick model for the local-minimum geometries of our sample molecules are shown in Figure 4.

In order to compare with astronomical spectra, we apply the Drude profiles to the molecules, creating broad emission bands from the vibrational lines. The normal mode vibrational line(s) that coincides with the peaks of the emission band are used to classify and label the band. We find that in a majority of the cases, the most intense IR lines under a particular emission band coincide well with the band peak position. For bands that overlap with intensity IR lines of similar strength, we rely on emission peak position to pick the corresponding vibrational mode.

The vibrational modes of the ovalene molecule in the 2-20 $\mu \mathrm{m}$ range are identified in Table 2 . Each vibrational mode is assigned based on our analysis of displacement vectors together with visual inspection of the animation of the vibrational modes. These results are generally consistent with usual interpretation of vibrations for aromatic/aliphatic compounds 


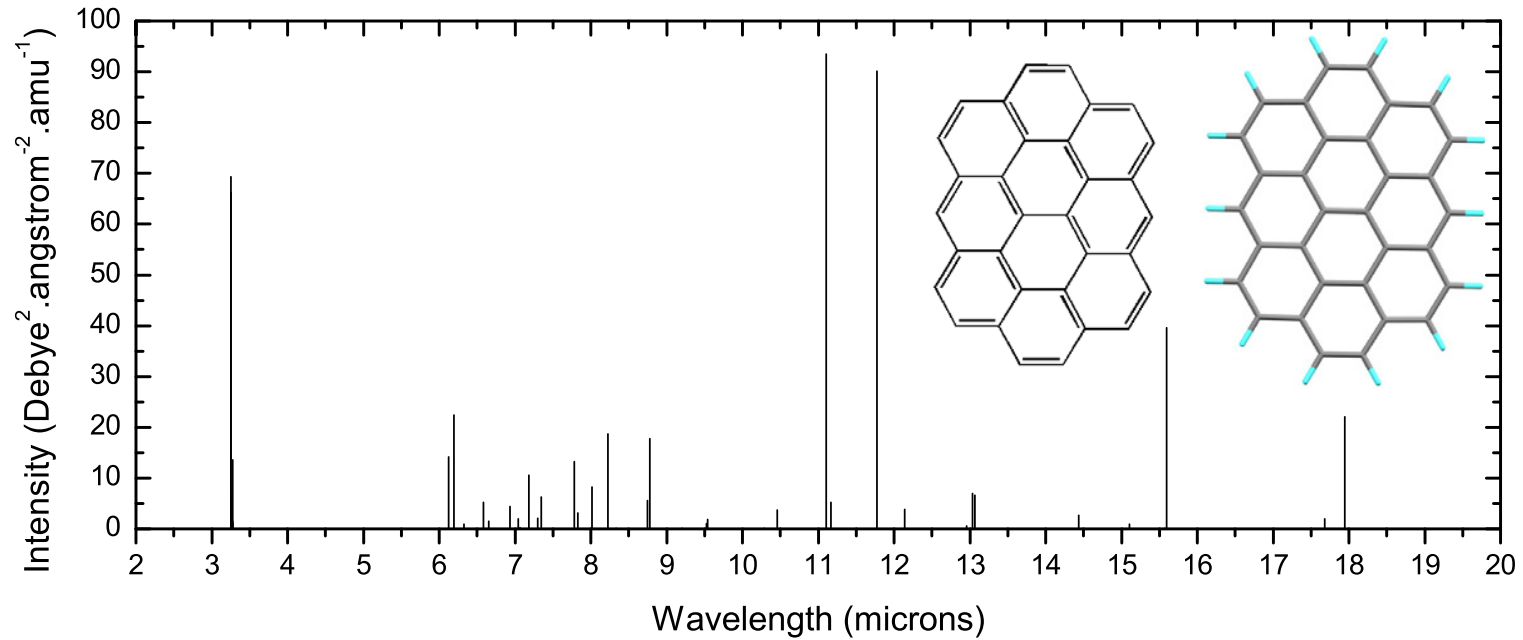

Figure 3. Calculated 2-20 $\mu \mathrm{m}$ spectrum of $\mathrm{C}_{32} \mathrm{H}_{14}$. The 2D structure and 3D capped stick representations of the optimized geometry of $\mathrm{C}_{32} \mathrm{H}_{14}$ are also shown.

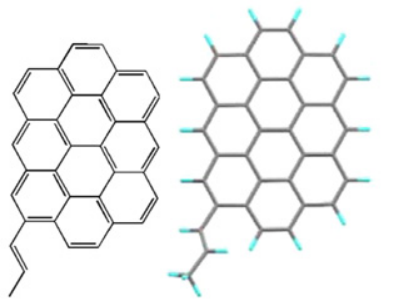

$\mathrm{C}_{35} \mathrm{H}_{18}$

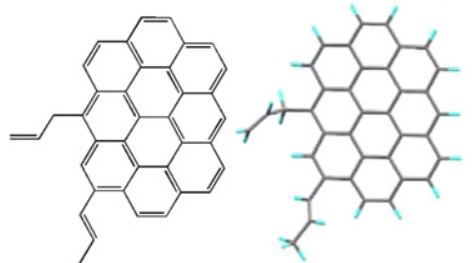

$\mathrm{C}_{38} \mathrm{H}_{22}$

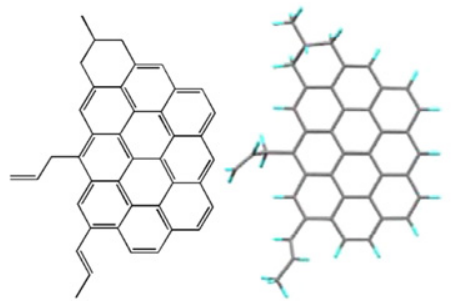

$\mathrm{C}_{42} \mathrm{H}_{28}$
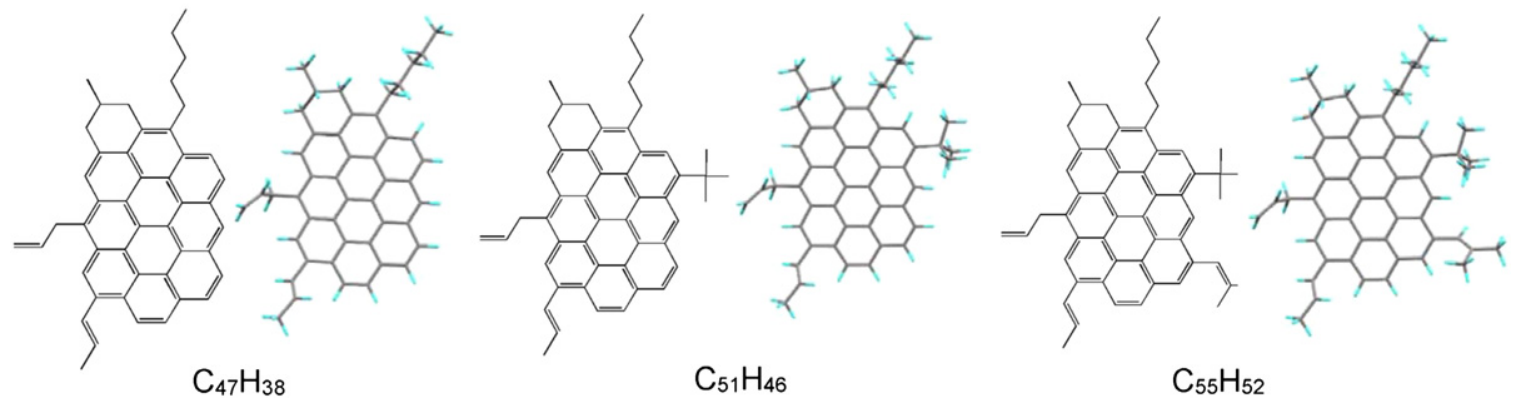

$\mathrm{C}_{55} \mathrm{H}_{52}$

Figure 4. 2D and 3D capped stick representations of the optimized geometry of our sample molecules. The molecules are (from left to right and from top to bottom) $\mathrm{C}_{35} \mathrm{H}_{18}, \mathrm{C}_{38} \mathrm{H}_{22}, \mathrm{C}_{42} \mathrm{H}_{28}, \mathrm{C}_{47} \mathrm{H}_{38}, \mathrm{C}_{51} \mathrm{H}_{46}$, and $\mathrm{C}_{55} \mathrm{H}_{52}$.

Table 2

Identification of the Vibrational Modes of Emission Bands of Ovalene

\begin{tabular}{lc}
\hline \hline $\begin{array}{l}\text { Bands } \\
(\mu \mathrm{m})\end{array}$ & Vibrations \\
\hline 3.26 & $\mathrm{C}-\mathrm{H}$ stretching \\
6.18 & $\mathrm{C}=\mathrm{C}$ stretching coupled with in-plane $\mathrm{C}-\mathrm{H}$ bending \\
6.59 & $\mathrm{C}=\mathrm{C}$ stretching coupled with in-plane $\mathrm{C}-\mathrm{H}$ bending \\
7.19 & $\mathrm{C}=\mathrm{C}$ stretching coupled with in-plane C-H bending \\
7.80 & $\mathrm{C}=\mathrm{C}$ stretching coupled with in-plane C-H bending \\
8.22 & $\mathrm{C}-\mathrm{H}$ in-plane bending \\
8.77 & $\mathrm{C}-\mathrm{H}$ in-plane bending \\
9.54 & {$[\mathrm{C}=\mathrm{C}]$ in-plane rocking mode unit coupled with in-plane C-H bending } \\
11.11 & $\mathrm{C}-\mathrm{H}$ out-of-plane bending coupled with out-of-plane ring deformation \\
11.78 & $\mathrm{C}-\mathrm{H}$ out-of-plane bending \\
13.04 & $\mathrm{C}-\mathrm{H}$ out-of-plane bending/in-plane ring deformation \\
14.14 & In-plane ring deformation \\
15.59 & $\mathrm{C}-\mathrm{H}$ out-of-plane bending coupled with out-of-plane ring deformation \\
17.94 & $\mathrm{C}-\mathrm{H}$ out-of-plane bending coupled with out-of-plane ring deformation
\end{tabular}

(Duley \& Williams 1983). For example, the $3.3 \mu \mathrm{m}$ band is assigned as an aromatic $\mathrm{C}-\mathrm{H}$ stretch. However, some other bands are the result of coupled motions and their vibrational mode assignments are not as straightforward as the $3.3 \mu \mathrm{m}$ feature.
Figures 5-10 show the spectral changes as aliphatic components are added to the ovalene molecule. A glance at these figures shows that the PAH bands at $8.77 \mu \mathrm{m}, 11.78 \mu \mathrm{m}, 13.04 \mu \mathrm{m}$, $15.59 \mu \mathrm{m}$, and $17.94 \mu \mathrm{m}$ weaken relatively upon the addition of aliphatic groups. Qualitatively, all of these bands show the contribution from in-plane or out-of-plane bending vibrations of aromatic $\mathrm{C}-\mathrm{H}$ bonds (Table 2). The weakening of these bands is the result of replacing these hydrogens by aliphatic groups.

Below, we discuss in more detail the spectra in separate wavelength regions.

1. 3-4 $\mu \mathrm{m}$ region (Figure 5). The ovalene molecule has a single emission band at $3.25 \mu \mathrm{m}$ due to aromatic $\mathrm{C}-\mathrm{H}$ stretch. When aliphatic groups are added, a broader feature around $3.45 \mu \mathrm{m}$ begins to appear. Vibrational analysis shows that this band is due to aliphatic $\left(s p^{3}\right) \mathrm{C}-\mathrm{H}$ stretching modes. The addition of more aliphatic groups causes the two bands to merge, as can be seen in the spectrum of $\mathrm{C}_{55} \mathrm{H}_{52}$. These results are in agreement with the functional group frequency assignment scheme widely used in organic chemistry (Fuhrer et al. 1972; Günzler \& Gremlich 2002). 


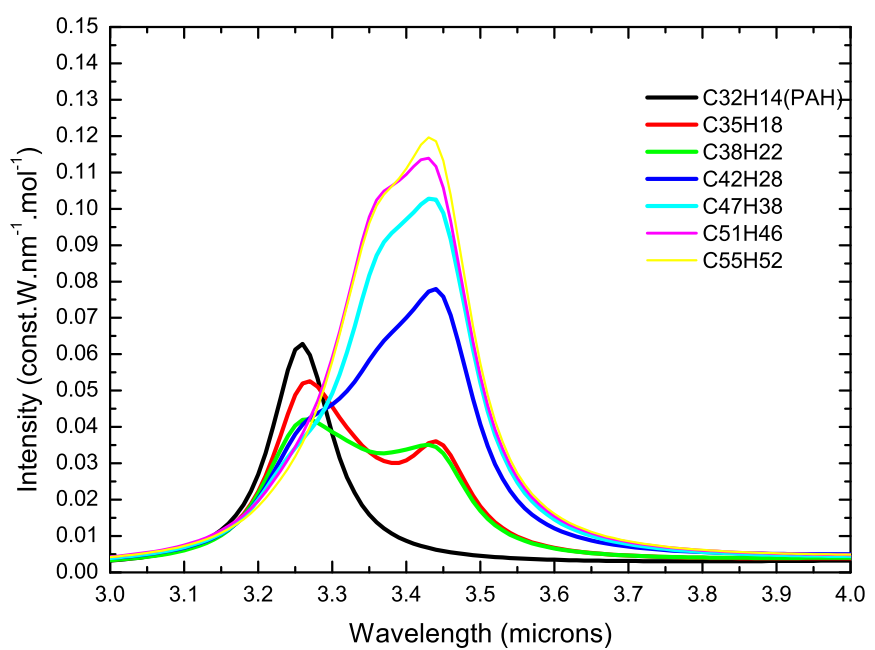

Figure 5. Simulated 3.0-4.0 $\mu \mathrm{m}$ emission spectra of the seven molecules with increasing degrees of aliphatic structures. The first molecule $\mathrm{C}_{32} \mathrm{H}_{14}$ (ovalene) is a purely aromatic molecule.

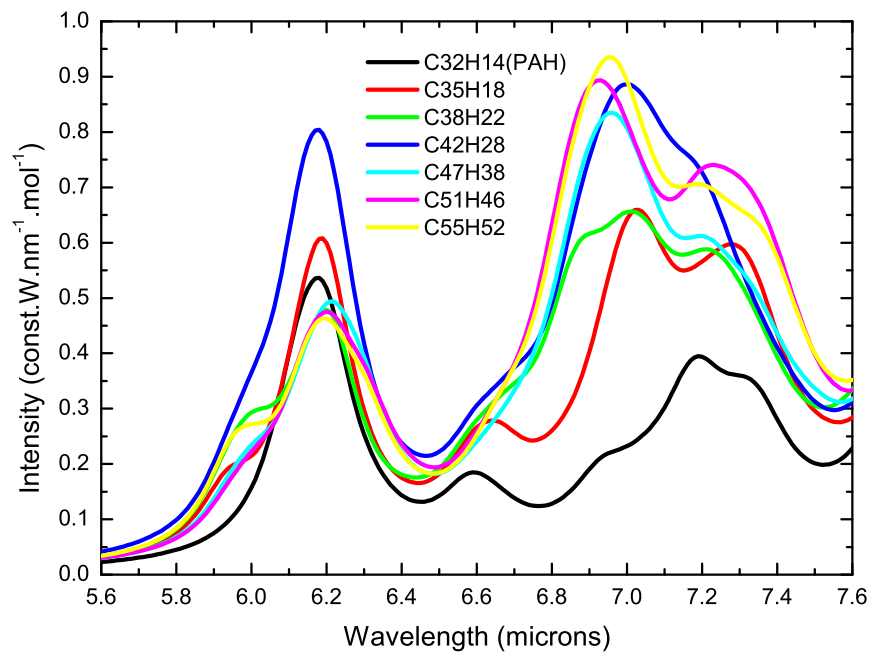

Figure 6. Simulated 5.6-7.6 $\mu \mathrm{m}$ emission spectra of the seven molecules with increasing degrees of aliphatic structures.

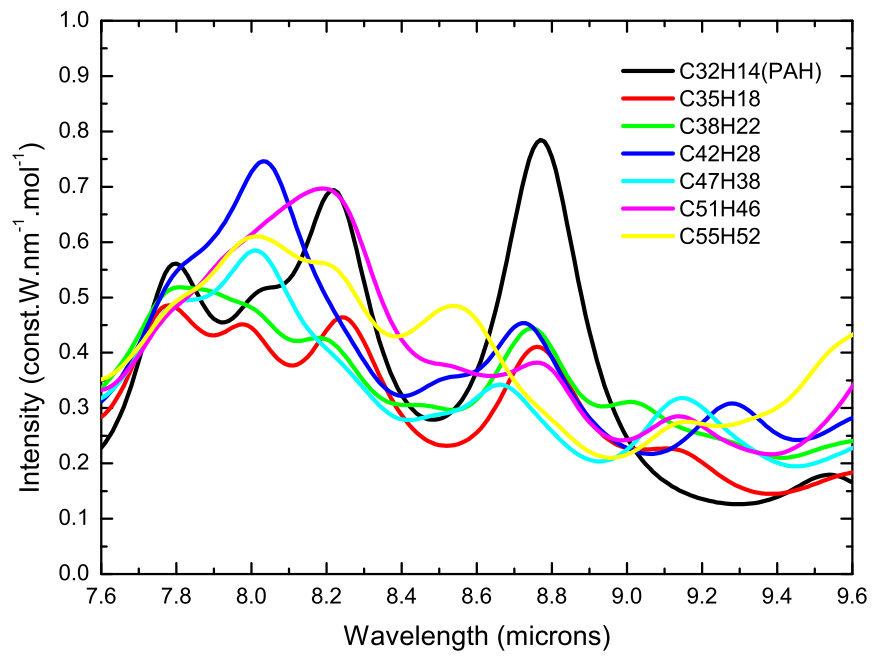

Figure 7. Simulated 7.6-9.6 $\mu \mathrm{m}$ emission spectra of the seven molecules with increasing degrees of aliphatic structures.

2. 5.6-7.6 $\mu \mathrm{m}$ region (Figure 6). The $6.2 \mu \mathrm{m}$ band is a common feature among all the molecules under study. Normal mode analysis shows that this band is due to aromatic $\mathrm{C}=\mathrm{C}$ stretching coupled with the in-plane bending

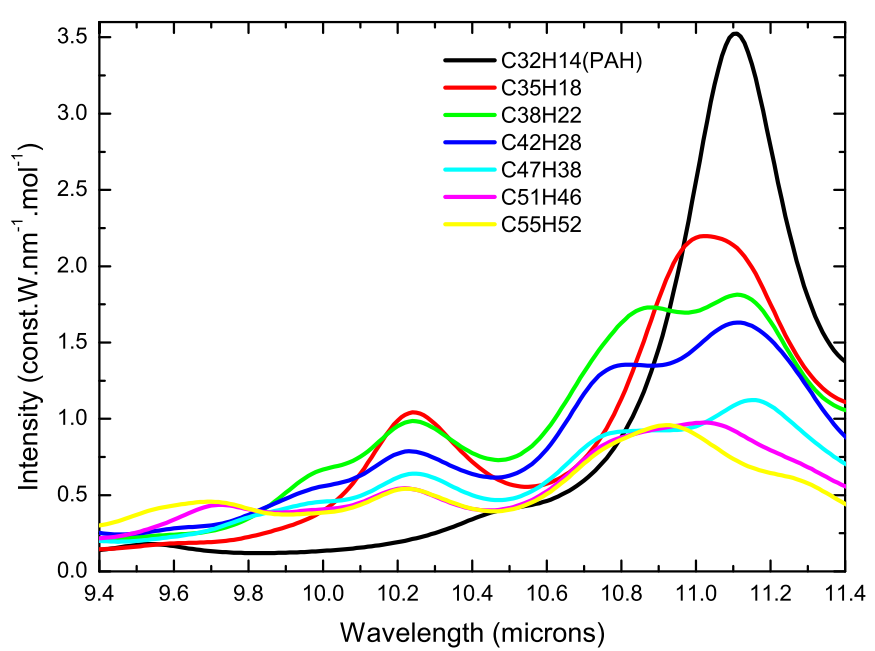

Figure 8. Simulated 9.4-11.4 $\mu \mathrm{m}$ emission spectra of the seven molecules with increasing degrees of aliphatic structures.

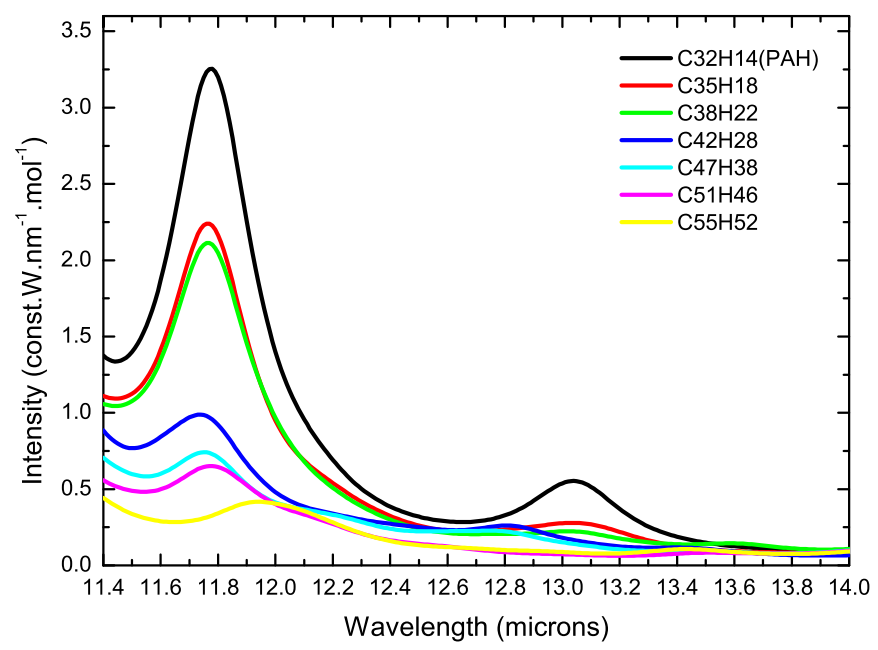

Figure 9. Simulated 11.4-14.0 $\mu \mathrm{m}$ emission spectra of the seven molecules with increasing degrees of aliphatic structures.

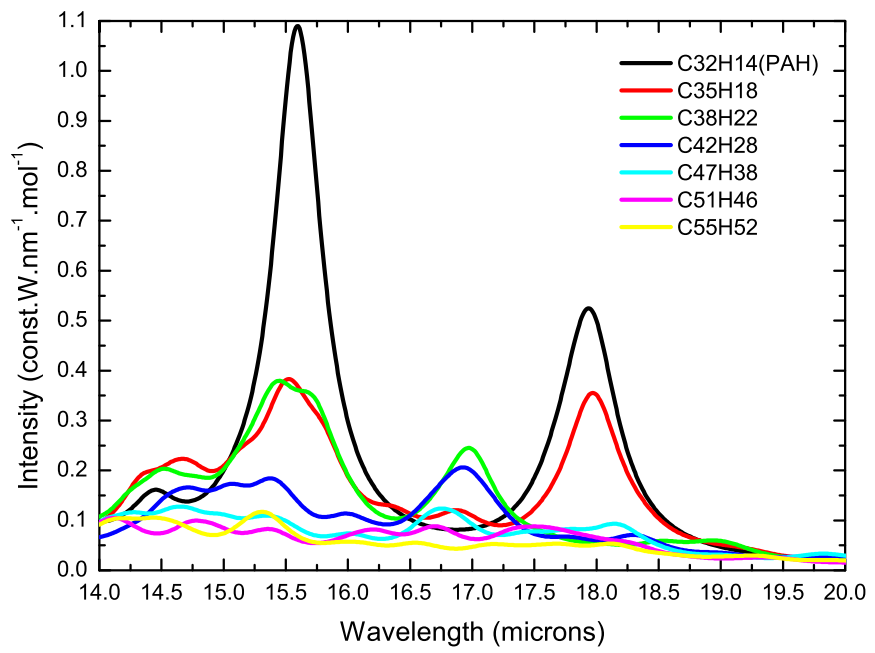

Figure 10. Simulated 14.0-20.0 $\mu \mathrm{m}$ emission spectra of the seven molecules with increasing degrees of aliphatic structures.

mode of aromatic $\mathrm{C}-\mathrm{H}$ bonds. An aliphatic feature is observed as a weak peak at $6.01 \mu \mathrm{m}$ at the shoulder to the aromatic $6.2 \mu \mathrm{m}$ band. This $6.01 \mu \mathrm{m}$ feature can be identified as aliphatic $\mathrm{C}=\mathrm{C}$ stretching coupled with 
aliphatic $=\mathrm{C}-\mathrm{H}$ in-plane bending modes found in the $\mathrm{H}_{2} \mathrm{C}=\mathrm{CH}-\mathrm{CH}_{2}-$ and $\mathrm{H}_{3} \mathrm{C}-\mathrm{CH}=\mathrm{CH}-$ functional groups. This band is blueshifted to $5.94 \mu \mathrm{m}$ when the aliphatic double bond is conjugated to an aromatic skeleton $\left(\mathrm{C}_{38} \mathrm{H}_{22}\right)$. In this case, the carbon atom of the ring bearing the aliphatic group also possesses considerable displacement, and thus our normal mode analysis counts this motion as one of the mixed modes $\left(\mathrm{C}_{38} \mathrm{H}_{22}\right)$. Ovalene (PAH) shows a weak band at $6.59 \mu \mathrm{m}$ composed of coupled in-plane $\mathrm{C}=\mathrm{C}$ stretching and $\mathrm{C}-\mathrm{H}$ bending vibrations (Table 2). The band shifts to $6.64 \mu \mathrm{m}$ upon replacement of the first hydrogen atom by the $\mathrm{H}_{3} \mathrm{C}-\mathrm{CH}=\mathrm{CH}$-group $\left(\mathrm{C}_{35} \mathrm{H}_{18}\right)$. This band vanishes completely in all other molecules in our series.

Aliphatic group vibrational modes split the ovalene emission band at $7.19 \mu \mathrm{m}$ into two bands. One centered at $7 \mu \mathrm{m}$ and the other band at $7.25 \mu \mathrm{m}$. The vibrations at $7 \mu \mathrm{m}$ are found to be deformation motions of $-\mathrm{CH}_{3}$ (methyl) groups and scissoring motions of aliphatic $-\mathrm{CH}_{2}$ (methylene) groups (Günzler \& Gremlich 2002). These aliphatic motions combine with aromatic vibrational modes at this region to form the $7.25 \mu \mathrm{m}$ band. The addition of more aliphatic $-\mathrm{CH}_{3}$ and $-\mathrm{CH}_{2}$ groups results in a merging of these two bands $(7 \mu \mathrm{m}$ and $7.25 \mu \mathrm{m})$ and the formation of the broadened band at $6.95 \mu \mathrm{m}\left(\mathrm{C}_{55} \mathrm{H}_{52}\right)$. This is also supported by the increase in the number of aliphatic and mixed modes as calculated via our normal mode decomposition analysis.

3. 7.6-9.6 $\mu \mathrm{m}$ region (Figure 7). In this spectral region, we see a marked growth in the number of pure aliphatic and mixed modes. As aliphatic groups are added to the ovalene molecule, three distinct bands of ovalene at 7.80, 8.22, and $8.77 \mu \mathrm{m}$ merge together and appear as one broad feature at around $8 \mu \mathrm{m}$ in $\mathrm{C}_{55} \mathrm{H}_{52}$. Our vibrational analysis shows that the coupling of $-\mathrm{CH}_{2}$ groups twisting vibrational modes (Günzler \& Gremlich 2002) with the aromatic vibrations at this region is the main reason for this change. Other types of aliphatic motions include $\mathrm{H}-\mathrm{C}=\mathrm{C}-\mathrm{H}$ (para, inplane bending), $-\mathrm{CH}_{3}$ deformation, and $-\mathrm{C}-\mathrm{H}$ (aromatic, bending) modes. The strong $8.77 \mu \mathrm{m}$ band in ovalene is blueshifted gradually upon combining with aliphatic vibrations and ultimately appears as a weak band around $8.54 \mu \mathrm{m}$ in $\mathrm{C}_{55} \mathrm{H}_{52}$.

The vibration under the weak $9.54 \mu \mathrm{m}$ band of ovalene cannot simply be assigned by the usual terminology. We suggest using the in-plane rocking mode of aromatic $\mathrm{HC}=\mathrm{CH}$ unit for labeling this vibration. This band is replaced by the weak band in the range of $9.1-9.3 \mu \mathrm{m}$ as a result of combination with $-\mathrm{CH}_{3}$ and $-\mathrm{CH}_{2}$ deformation, wagging, and twisting modes. The band intensity $(9.1-9.3 \mu \mathrm{m})$ increases when these aliphatic groups are added in the form of a cyclohexane group $\left(\mathrm{C}_{47} \mathrm{H}_{36}\right)$.

4. 9.4-11.4 $\mu \mathrm{m}$ region (Figure 8). $\mathrm{C}_{55} \mathrm{H}_{52}$, the molecule with the largest number of aliphatic groups, shows a weak band at $9.7 \mu \mathrm{m}$. This band can be identified as coupling between various twisting/wagging modes of $-\mathrm{CH}_{2}$ groups and the deformation mode of $-\mathrm{CH}_{3}$ groups and aromatic in-plane $\mathrm{C}-\mathrm{H}$ bending modes. The vibrations of aromatic $\mathrm{C}-\mathrm{H}$ bonds are different from what we observed in the ovalene molecule at $9.54 \mu \mathrm{m}$, which weakens with the addition of aliphatic groups.

The out-of-plane bending modes of para-hydrogens in the $\mathrm{H}_{3} \mathrm{CHC}=\mathrm{CH}-$ group results in the appearance of a band at $10.24 \mu \mathrm{m}$ (see the spectrum of $\mathrm{C}_{35} \mathrm{H}_{18}$ ). This feature exists for all of the molecules in our series. One of the other characteristic vibrations that appears under the $10.24 \mu \mathrm{m}$ band is the stretching mode of the $\mathrm{C}-\mathrm{C}$ single bond in the pentane side chain.

The most notable feature in this spectral region is the strong band of ovalene (PAH) at $11.11 \mu \mathrm{m}$ (Table 2). The combination of aromatic $-\mathrm{C}-\mathrm{H}$ out-of-plane bending modes with deformation vibrations of $-\mathrm{CH}_{3}$ groups shifts this strong band to the $11.03 \mu \mathrm{m}$ band, as can be seen in the spectrum of the $\mathrm{C}_{35} \mathrm{H}_{18}$ molecule. The addition of the $\mathrm{H}_{2} \mathrm{C}=\mathrm{CHCH}_{2}-$ aliphatic group causes the formation of another band at $10.85 \mu \mathrm{m}$. The aliphatic vibrations under this band are out-of-plane bending modes of olefin $\mathrm{C}-\mathrm{H}$ bonds $\left(\mathrm{H}_{2} \mathrm{C}=\right)$. These vibrations are coupled with the out-of-plane bending modes of aromatic $\mathrm{C}-\mathrm{H}$ bonds. By increasing the number of $-\mathrm{CH}_{3}$ and $-\mathrm{CH}_{2}$ groups, the deformation modes of $-\mathrm{CH}_{3}$ groups and twisting or rocking modes of $-\mathrm{CH}_{2}$ groups are added to the olefinic vibrations in this region of the spectra. The coupling between all of these aliphatic modes and the aromatic out-of-plane $\mathrm{C}-\mathrm{H}$ bending modes results in the replacement of the strong $11.11 \mu \mathrm{m}$ feature in ovalene with the $10.92 \mu \mathrm{m}$ broadband in $\mathrm{C}_{55} \mathrm{H}_{52}$.

5. $11.4-14.0 \mu \mathrm{m}$ region (Figure 9). The strong $11.78 \mu \mathrm{m}$ band (Table 2) in ovalene is gradually weakened by the decrease in the number of aromatic $\mathrm{C}-\mathrm{H}$ bonds in the other molecules. At the same time, the band position is redshifted to $12 \mu \mathrm{m}$ by combining the $\mathrm{C}-\mathrm{H}$ out-of-plane bending mode with the wagging and deformation modes of $-\mathrm{CH}_{2}$ and $-\mathrm{CH}_{3}$, especially the methyl and methylene groups on the cyclohexane ring. The coupling of various $\mathrm{C}-\mathrm{C}-\mathrm{C}^{\text {aromatic }}$ bond angle deformation modes of aliphatic groups, for instance, $\mathrm{H}_{2} \mathrm{C}=\mathrm{CH}-\mathrm{CH}_{2}-\mathrm{C}^{\text {aromatic }}$ with the PAH modes at $13.04 \mu \mathrm{m}$, forms a very weak band around $13.34 \mu \mathrm{m}$ in $\mathrm{C}_{55} \mathrm{H}_{52}$. This feature can hardly be seen in Figure 9.

6. $14.0-20.0 \mu \mathrm{m}$ region (Figure 10). While the bands of ovalene $(\mathrm{PAH})$ can be identified by the usual vibrational modes (Figure 11), the bands of the other molecules in our series in this part of the spectrum cannot be so easily labeled (Figure 12). Our simple displacement vector analysis shows that the motions involve a large fraction of the molecules and are more complex in nature. However, distinction between aromatic and aliphatic contributions can still be made. The ovalene (PAH) molecule has a weak band at $14.45 \mu \mathrm{m}$ and two strong bands at $15.59 \mu \mathrm{m}$ and $17.94 \mu \mathrm{m}$. These bands are weakened by the replacement of aromatic $\mathrm{C}-\mathrm{H}$ bonds with aliphatic groups. The only noticeable fingerprint of the aliphatic group in this region is the concerted rocking motion of $-\mathrm{CH}_{2}$ groups on the pentane side chain. This vibration forms a band at $15.31 \mu \mathrm{m}$ in $\mathrm{C}_{55} \mathrm{H}_{52}$. Based on our analysis, it is suggested that the strong $17.94 \mu \mathrm{m}$ band (Table 2) in ovalene is blueshifted to the $16.9 \mu \mathrm{m}$ region and then gradually vanishes.

\section{DISCUSSIONS}

The UIE phenomenon is a complex one. In addition to the aromatic features at $3.3,6.2,7.7,8.6$, and $11.3 \mu \mathrm{m}$, there are aliphatic features at 3.4 and $6.9 \mu \mathrm{m}$. There are also unidentified features at 15.8, 16.4, 17.4, 17.8, and $18.9 \mu \mathrm{m}$ which are observed in protoplanetary nebulae (Kwok et al. 1999), reflection nebulae (Sellgren, Uchida \& Werner 2007), and galaxies (Sturm et al. 2000). Broad emission plateau features at 8 and 


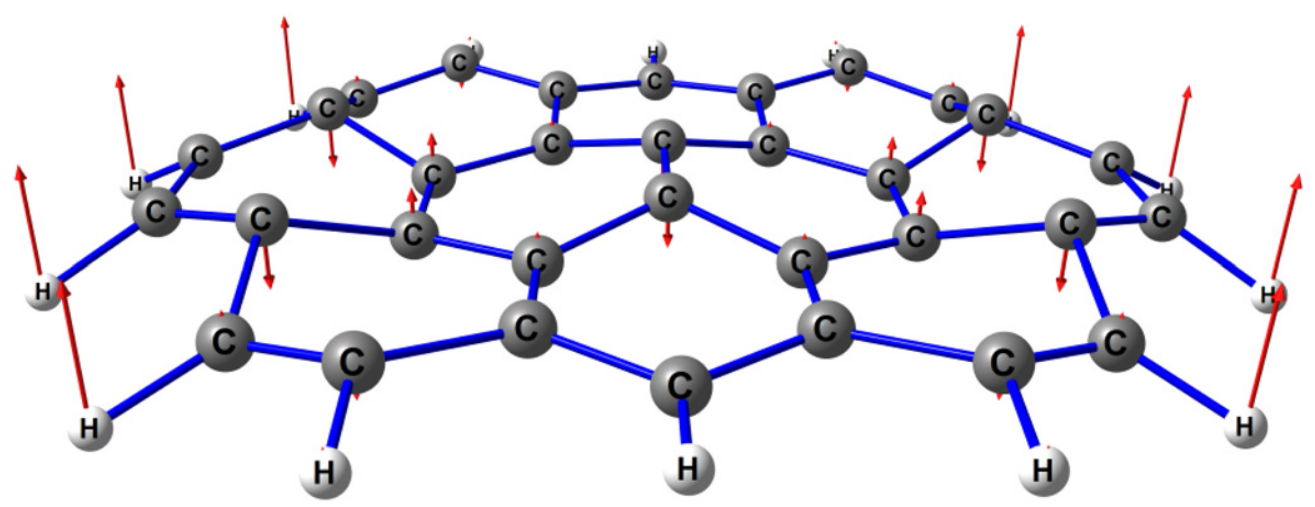

Figure 11. Snapshot of animation showing the vibrational motion associated with the $15.59 \mu \mathrm{m}$ feature of ovalene $\left(\mathrm{C}_{32} \mathrm{H}_{14}\right)$. The direction and magnitude of motions are indicated by arrows.

(An animation of this figure is available).

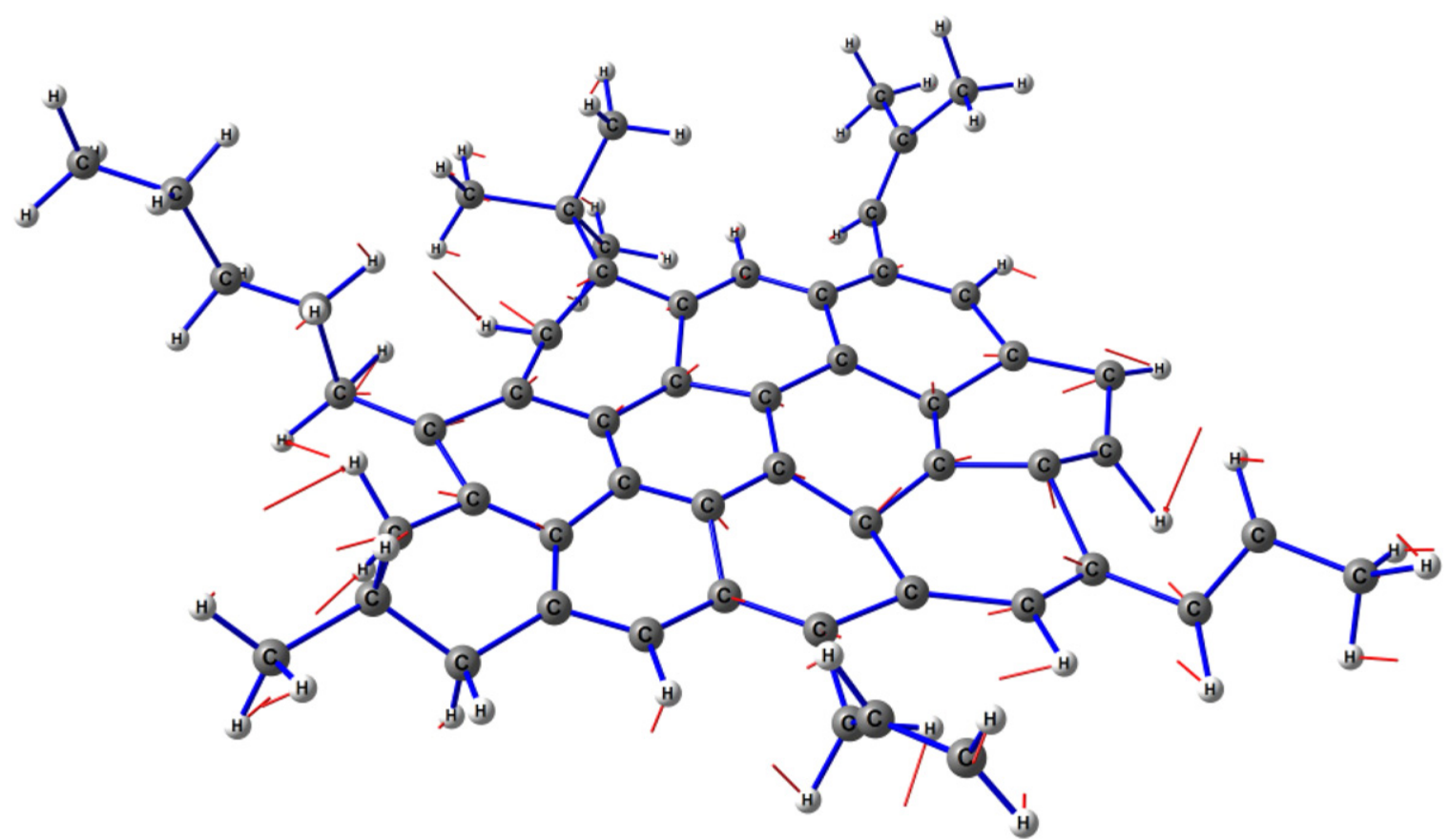

Figure 12. Snapshot of animation showing the vibrational motion associated with the $15.53 \mu \mathrm{m}$ feature of $\mathrm{C}_{55} \mathrm{H}_{52}$. The direction and magnitude of motions are indicated by arrows.

(An animation of this figure is available).

$12 \mu \mathrm{m}$, and maybe also around $17 \mu \mathrm{m}$, are commonly observed. In recent years, there has been increased interest in the plateau features, as they are found to be associated with the presence of fullerene $\left(\mathrm{C}_{60}\right)$ (Zhang \& Kwok 2013; Otsuka et al. 2014). The unidentified $21 \mu \mathrm{m}$ feature (Kwok et al. 1989) is also often accompanied by the 8 and $12 \mu \mathrm{m}$ plateau features (Cerrigone et al. 2011; Volk et al. 2011). Suggestions have been made that the carrier of the plateau features are precursors to fullerene (Bernard-Salas et al. 2012; García-Hernández et al. 2012). A good understanding of the origin of the plateau features is key to the identification of the chemical structure of the carrier of the UIE bands and the history of chemical synthesis in the late stages of stellar evolution.

How well can a mixed aromatic/aliphatic model work in simulating the astronomical UIE bands in comparison to the PAH model? Traditionally, the UIE bands are explained by collective emissions from a mixture of PAH molecules. Cook \& Saykally (1998) used a family of nine neutral PAHs (including ovalene) to simulate the astronomical spectra. They found that the inclusion of cation PAHs can explain the UIE better than a collection of neutral PAHs, but their simulation still cannot reproduce the details of UIE. They suggest that a larger number of PAHs and/or larger PAHs are required. With data on hundreds of PAH molecules now available from the NASA Ames PAH database (Boersma et al. 2014), it is possible to use PAH molecules with different sizes and charged states to simulate the astronomical UIE spectra (Cami 2011). However, questions have been raised concerning how meaningful such fitting exercises are due to the large number of parameters involved (Zhang \& Kwok 2015).

Although the range of mixed aromatic/aliphatic structures that we have calculated is limited, Figure $13\left(\mathrm{C}_{55} \mathrm{H}_{52}\right.$, an ovalene molecule with aliphatic branches) shows that aliphatic side groups can also create a host of features in the 6-9 and 10-14 $\mu \mathrm{m}$ regions, and therefore offers the possibility of explaining the 8 and $12 \mu \mathrm{m}$ plateau emission features. In the $15-20 \mu \mathrm{m}$ region, 


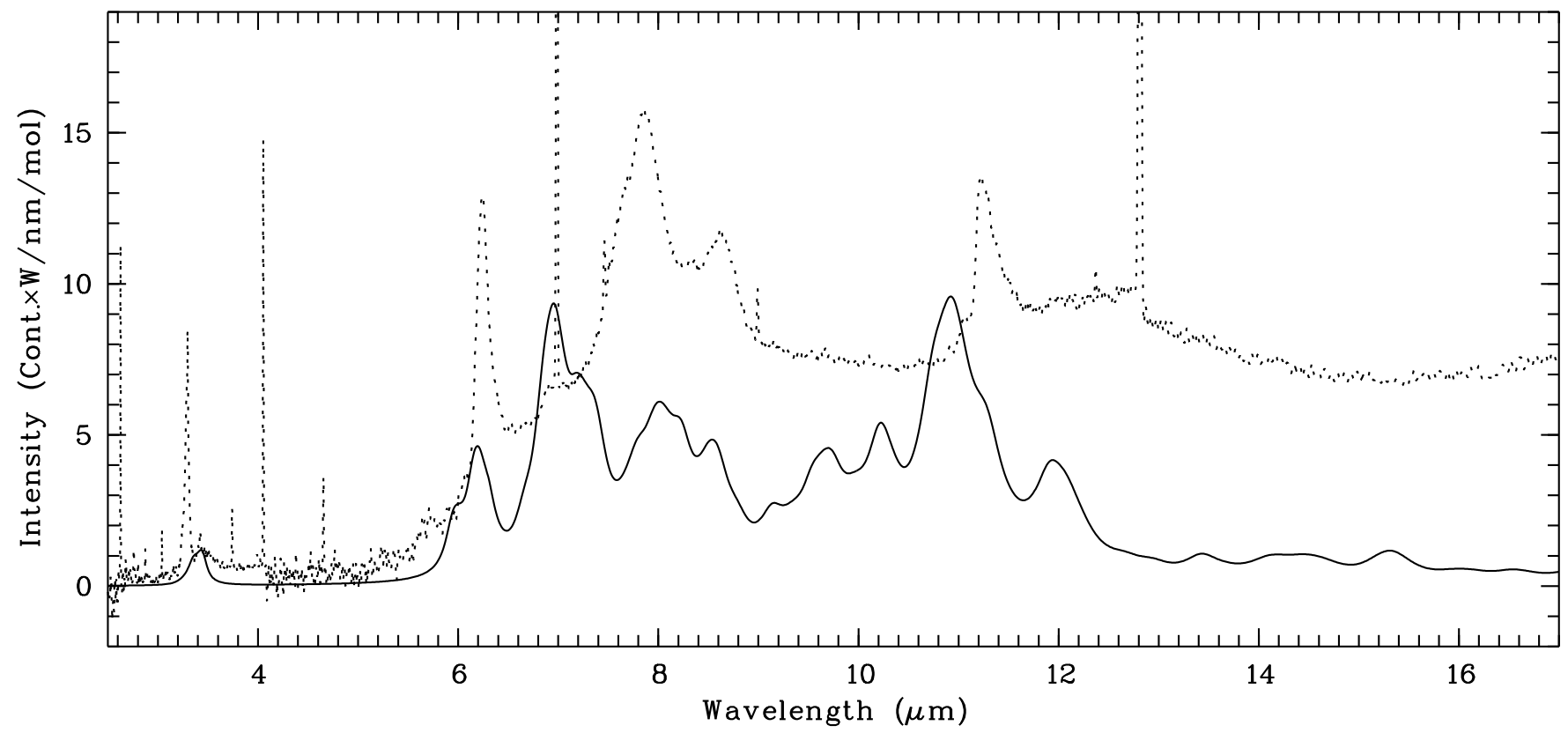

Figure 13. Comparison of the simulated emission spectrum of $\mathrm{C}_{55} \mathrm{H}_{52}$ (solid line) with the Infrared Space Observatory spectrum (dotted line) of the planetary nebula $\mathrm{BD}+30^{\circ} 3639$ (in units of $F_{\lambda}$ ). The narrow features in the astronomical spectrum are atomic lines.

the mixed aromatic/aliphatic structure can lead to complex vibrational motions, and could form the basis to explaining the $17 \mu \mathrm{m}$ plateau emission feature. These results suggest that the inclusion of aliphatic side groups will help our interpretation of the UIE phenomenon.

\section{CONCLUSIONS}

We have conducted a theoretical study on the changes in the infrared emission spectra of the PAH molecule ovalene through the replacement of aromatic hydrogen atoms by aliphatic groups. The local-minimum geometry and the corresponding vibrational normal modes are calculated by applying the DFT. The IR absorption line positions are then corrected for anharmonicity, couplings, and other complex electronic effects by applying the most recently developed vibrational scale factor scheme. In order make comparisons with astronomical spectra, these absorption lines are translated to emission bands assuming a Drude profile and thermal excitation. The resulting emission spectra show good agreement with available laboratory experimental data.

In order to understand the type of vibrational motions associated with each simulated emission bands, the calculated absorption lines are mapped back to the corresponding simulated emission spectra. Through the use of a simple quantitative analysis of cartesian components of the displacement vector of all atoms for each normal mode (IR or Raman active), we are able to separate the contributions of aromatic and aliphatic parts. Results show that the major regions of IR spectra affected by aliphatic groups are 3-4, 6-7, 7-8, and 8-9 $\mu \mathrm{m}$. Among the last three regions, the region of 7-8 $\mu \mathrm{m}$ contains the largest number of aliphatic vibrations. This is the region of spectra where $8 \mu \mathrm{m}$ broad plateau feature is observed in astronomical sources and we suggest that aliphatic side groups play a major role in the formation of the $8 \mu \mathrm{m}$ plateau feature.

The theoretical scheme described in this work can be very useful in the interpretation of astronomical spectra and the identification of the structure of the molecular carrier of the
UIE bands. In this paper, we have limited ourselves to the study of molecules with an aromatic core and aliphatic branches. In order to further explore the MAON model, we will study more irregular structures with random aromatic rings connected by randomly oriented aliphatic branches. Since such structures are more complex, larger computational resources are required. We hope this work will motivate further theoretical studies of large, amorphous organic compounds for a better understanding of the origin of the astronomical UIE bands.

This work was partially supported by the Research Grants Council of the Hong Kong Special Administrative Region, China (project No. HKU 7031/10P).

\section{REFERENCES}

Bauschlicher, C. W., Jr., Boersma, C., Ricca, A., et al. 2010, ApJS, 189, 341 Bauschlicher, C. W., \& Langhoff, S. R. 1997, AcSpA, 53, 1225 Becke, A. D. 1993, JChPh, 98, 1372

Bernard-Salas, J., Cami, J., Peeters, E., et al. 2012, ApJ, 757, 41 Boersma, C., Bauschlicher, C. W., Jr., Ricca, A., et al. 2014, ApJS, 211, 8 Born, M., \& Oppenheimer, R. 1927, AnP, 389, 457

Buss, R. H., Cohen, M., Tielens, A. G. G. M., et al. 1990, ApJL, 365, L23 Cami, J. 2011, in PAHs and the Universe, ed. C. Joblin \& A. G. G. M. Tielens (EAS Publications Ser. 46; Les Ulis: EDP Sciences), 117

Cerrigone, L., Hora, J. L., Umana, G., et al. 2011, ApJ, 738, 121

Chiar, J. E., Adamson, A. J., Pendleton, Y. J., et al. 2002, ApJ, 570, 198

Cook, D. J., \& Saykally, R. J. 1998, ApJ, 493, 793

Cook, D. J., Schlemmer, S., Balucani, N., et al. 1996, Natur, 380, 227

Cook, D. J., Schlemmer, S., Balucani, N., et al. 1998, JPhChA, 102, 1465

Dartois, E. 2011, in PAHs and the Universe, ed. C. Joblin \& A. G. G. M. Tielens (EAS Publications Ser. 46; Les Ulis: EDP Sciences), 381

Dartois, E., Geballe, T., Pino, T., et al. 2007, A\&A, 463, 635

Dartois, E., Muñoz Caro, G. M., Deboffle, D., \& d'Hendecourt, L. 2004, A\&A, 423, L33

Dischler, B., Bubenzer, A., \& Koidl, P. 1983a, SSCom, 48, 105

Dischler, B., Bubenzer, A., \& Koidl, P. 1983b, ApPhL, 42, 636

Duley, W. W., \& Williams, D. A. 1983, MNRAS, 205, 67

Frisch, M. J., Frisch, G. W., Trucks, H. B., et al. 2009, Gaussian 09, Revision, C.01 (Wallingford, CT: Gaussian, Inc.)

Fuhrer, H., Kartha, V. B., Krueger, P. J., Mantsch, H. H., \& Jones, R. N. 1972, Chem. Rev., 72, 439 
García-Hernández, D. A., Villaver, E., Garca-Lario, P., et al. 2012, ApJ, 760,107

Geballe, T. R., Tielens, A. G. G. M., Kwok, S., \& Hrivnak, B. J. 1992, ApJL, 387, L89

Günzler, H., \& Gremlich, H. U. 2002, in IR Spectrosocopy (An Introduction) ed. M.-J. Blümich (New York: Wiley)

Hohenberg, P., \& Kohn, W. 1964, PhR, 136, B864

Hrivnak, B. J., Geballe, T. R., \& Kwok, S. 2007, ApJ, 662, 1059

Jensen, F. 2001, JChPh, 115, 9113

Jensen, F. 2002, JChPh, 116, 7372

Jones, A. P. 2012a, A\&A, 540, A2

Jones, A. P. 2012b, A\&A, 540, A1

Jones, A. P. 2012c, A\&A, 545, C3

Jourdain de Muizon, M., D’Hendecourt, L. B., \& Geballe, T. R. 1990, A\&A, 235,367

Kohn, W., \& Sham, L. J. 1965, PhR, 140, A1133

Kwok, S., Volk, K., \& Bernath, P. 2001, ApJL, 554, L87

Kwok, S., Volk, K. M., \& Hrivnak, B. J. 1989, ApJL, 345, L51

Kwok, S., Volk, K., \& Hrivnak, B. J. 1999, A\&A, 350, L35

Kwok, S., \& Zhang, Y. 2011, Natur, 479, 80

Laury, M. L., Carlson, M. J., \& Wilson, A. K. 2012, JCoCh, 33, 2380

Malloci, G., Joblin, C., \& Mulas, G. 2007, CP, 332, 353

Ochterski, J. W. 1999, Vibrational Analysis in Gaussian (Guassian White Papers), www.Gaussian.com
Otsuka, M., Kemper, F., Cami, J., Peeters, E., \& Bernard-Salas, J. 2014, MNRAS, 437, 2577

Papoular, R. 2013, MNRAS, 434, 862

Papoular, R. J., \& Papoular, R. 2014, MNRAS, 443, 2974

Ricca, A., Bauschlicher, C. W., Jr., \& Allamandola, L. J. 2013, ApJ, 776,31

Robertson, J. 2002, Material Science and Engineering R, 37, 129

Sandford, S. A., Bernstein, M. P., \& Materese, C. K. 2013, ApJS, 205, 8

Sellgren, K., Uchida, K. I., \& Werner, M. W. 2007, ApJ, 659, 1338

Soifer, B. T., Russell, R. W., \& Merrill, K. M. 1976, ApJL, 207, L83

Sturm, E., Lutz, D., Tran, D., et al. 2000, A\&A, 358, 481

Volk, K., Hrivnak, B. J., Matsuura, M., et al. 2011, ApJ, 735, 127

Wickramasinghe, D. T., \& Allen, D. A. 1980, Natur, 287, 518

Willner, S. P., Puetter, R. C., Russell, R. W., \& Soifer, B. T. 1979, Ap\&SS, 65,95

Wright, G. S., Bridger, A., Geballe, T. R., \& Pendleton, Y. 1996, in Cold Dust and Galaxy Morphology, ed. D. L. Block \& J. M. Greenberg (Dordrecht: Kluwer), 143

Yamagishi, M., Kaneda, H., Ishihara, D., et al. 2012, A\&A, 541, A10

Yang, X. J., Glaser, R., Li, A., \& Zhong, J. X. 2013, ApJ, 776, 110

Zhang, Y., \& Kwok, S. 2013, EP\&S, 65, 1069

Zhang, Y., \& Kwok, S. 2015, ApJ, 798, 37

Ziegler, T. 1991, Chem. Rev., 91, 651 